\title{
Acute Peripheral Motor Neuropathy Induced by Oxaliplatin-Correlated Hypokalaemia
}

\author{
Riccardo Giampieri (D) - Elena Maccaroni · Valeria Sotte • \\ Maria G. Baleani - Tania Meletani · Enrica Giglio · Federica Pecci • \\ Alessandro Bittoni · Andrea Lanese - Luca Cantini · Giulia Mentrasti • \\ Alessandra Lucarelli · Rossana Berardi
}

Received: September 19, 2019 / Published online: December 5, 2019

(C) The Author(s) 2019

\section{ABSTRACT}

Neurotoxicity is one of the most common side effects of oxaliplatin-based therapy. Most patients who receive at least 3-4 months of treatment suffer from peripheral sensory neurotoxicity (PSN), characterised by the loss or impairment of tactile and proprioceptive sensory function. Motor impairment, such as muscle weakness or palsy, has been rarely described, and the physiopathology of PSN, as well as the motor symptoms due to oxaliplatinbased treatment, are not adequately understood. Here we report the case of a patient who experienced severe acute peripheral motor

Enhanced Digital Features To view enhanced digital features for this article, including a patient video, go to https://doi.org/10.6084/m9.figshare.10275383.

Electronic Supplementary Material The online version of this article (https://doi.org/10.1007/s40487019-00102-3) contains supplementary material, which is available to authorized users.

R. Giampieri (ه) · E. Maccaroni · V. Sotte ·

M. G. Baleani - T. Meletani - E. Giglio - F. Pecci .

A. Bittoni - A. Lanese - L. Cantini - G. Mentrasti .

A. Lucarelli · R. Berardi

Medical Oncology, University Hospital-Marche

Polytechnic University, Ancona, Italy

e-mail: riccardo.giampieri81@gmail.com neuropathy as a side effect of oxaliplatin-based treatment. We also review other cases of PSN published in the literature and suggest a novel hypothesis on the physiopathology of this particular event. Take-away lessons: Not all of the neurological symptoms observed during oxaliplatin-based treatment can be traced back directly to the oxaliplatin itself, and other factors, such as electrolyte imbalances, may contribute to the symptoms. Patients with gastrointestinal malignancies are the patients most affected by neurotoxicity due to the side effects of chemotherapy and the disease itself.

Keywords: Hypokalaemia; Neuropathy; Oxaliplatin 


\section{Key Summary Points}

Why carry out this study?

Oxaliplatin is used frequently to treat patients with gastrointestinal malignancies. The majority of patients treated with oxaliplatin develop peripheral sensory neurotoxicity (PSN) whereas motor impairment is seldom described.

This case report focusses on the occurrence of acute neurotoxicity in a patient treated with oxaliplatin. The patient showed signs of motor impairment in addition to standard PNS, suggesting a different explanation of the toxicity observed.

\section{What was learned from the study?}

The motor impairment seen in this patient was correlated to severe electrolyte imbalances, primarily in serum potassium levels, after oxaliplatin infusion.

This case suggests that adequate monitoring of potassium levels should be performed routinely. Furthermore, hypokalaemia could be more closely related to the glucose 5\% dilution rather than oxaliplatin itself.

\section{INTRODUCTION}

Oxaliplatin-based chemotherapy has rapidly evolved into one of the most widely used standard treatments for patients with primary gastrointestinal-derived malignancies. In particular, combinations of oxaliplatin and fluoropyrimidines are used in standard chemotherapy regimens for the treatment of colorectal [1-4], gastric [5] and pancreatic cancer patients [6]. However, oxaliplatin is, in a dose-dependent manner, also the main chemotherapeutic agent causing slowly

\section{CASE PRESENTATION}

\section{Patient Information}

Written informed consent for publication of this case and the patient's clinical details was requested and obtained from the patient himself and his family.

BA, a 64-year-old man, had recently undergone right hemicolectomy for primary colon 
cancer (stage pT3 pN1a cM0, stage IIIB sec. TNM AJCC-UICC 2009) at our hospital (University Hospital-Marche Polytechnic University, Anacona, Italy). XELOX adjuvant chemotherapy [13] was started, with eight cycles (over 6 months) of treatment planned, followed by clinical-radiological follow-up (as per institutional guidelines). The patient was not receiving any other investigational agents or incidental therapy for other relevant diseases at the time of treatment initiation.

The patient's family history was negative for significant morbidities, whereas his past medical history included brain surgery for a left temporal cavernous angioma 8 years prior to the diagnosis of colorectal cancer. Following this brain surgery, no significant changes were observed in the patient's behaviour, strength and memory. Brain magnetic resonance imaging scans were obtained as per the neurologist's suggestion as follow-up, with the last scan performed 3 months before colorectal surgery. The scan did not show any change that could suggest new brain disease.

The patient received the first two cycles of chemotherapy with acceptable toxicity. He complained of sensory neuropathy in the upper limbs, reporting a prickling/tingling sensation at the volar surface as well as in the fingers of the hand, both worsened by cold, that did not interfere with daily activities or function (National Cancer Institute Common Toxicity Criteria for Adverse Events [NCI CTCAE] Grade 1 neurotoxicity), for up to 14 days after the oxaliplatin infusion. He also complained of diarrhea (NCI CTCAE Grade 1) during the 14 days of capecitabine intake.

At the start of the third cycle of chemotherapy, the patient did not report any kind of sensory impairment, and his bowel function had returned to normal. Laboratory test results were around the range of normality with mild (3.1 $\mathrm{mEq} / \mathrm{l}$, not graded by NCI CTCAE) hypokalaemia (presumably due to the diarrhea) as the only altered result (Table 1). The patient was thus started on the infusion of chemotherapy with XELOX as shown in the timeline in Fig. 1, which also shows the relative doses of the drugs and infusion times.
Table 1 Patient serum electrolyte levels before the third cycle of chemotherapy

\begin{tabular}{lll}
\hline Electrolytes & $\begin{array}{l}\text { Laboratory test results } \\
\text { before third cycle of } \\
\text { chemotherapy }\end{array}$ & $\begin{array}{l}\text { Normal } \\
\text { electrolyte } \\
\text { range }\end{array}$ \\
\hline Potassium & $3.1 \mathrm{mEq} / \mathrm{l}$ & $3.6-4.9 \mathrm{mEq} / \mathrm{l}$ \\
Sodium & $143 \mathrm{mEq} / \mathrm{l}$ & $136-148 \mathrm{mEq} / 1$ \\
Calcium & $8.1 \mathrm{mg} / \mathrm{dl}$ & $8.5-10.5 \mathrm{mg} / \mathrm{dl}$ \\
Albumin & $3.8 \mathrm{~g} / \mathrm{dl}$ & $3.5-5 \mathrm{~g} / \mathrm{dl}$ \\
\hline
\end{tabular}

\section{Clinical Findings}

After oxaliplatin infusion (and the wash), the patient experienced severe neurological symptoms, including sudden myoclonic jerks in the muscles of the lower limbs, which occurred more frequently on the left side than on the right side, and muscle spasms in the upper hemisome. The neurological objective assessment reported slight hypostenia in the four limbs. The patient was able to perform grasping gestures with both hands, but his ability to release the grip in the left hand was severely affected (Electronic Supplementary Material Video 1). The patient also reported the sensation of a "sore throat", slight voice loss and breathing difficulty. He also complained of paraesthesia in the hands, which is a common side effect of oxaliplatin infusion and similar to those side effects he had experienced in previous chemotherapy cycles.

\section{Diagnostic Assessment}

At presentation of the first symptoms, we believed that a new episode of bleeding of the cavernous angioma had occurred. However, objective examination excluded signs related to such an event: the patient remained conscious at all times. Seizures were then ruled out as the cause. We then hypothesized that metabolic imbalance could be the causative factor, and the patient was re-tested for electrolytes, which revealed a significant drop in serum potassium levels (2.6 mEq/l; NCI CTCAE Grade 3).

\section{Therapeutic Intervention}

Following the discovery of low serum potassium levels, the patient was initially treated with a 


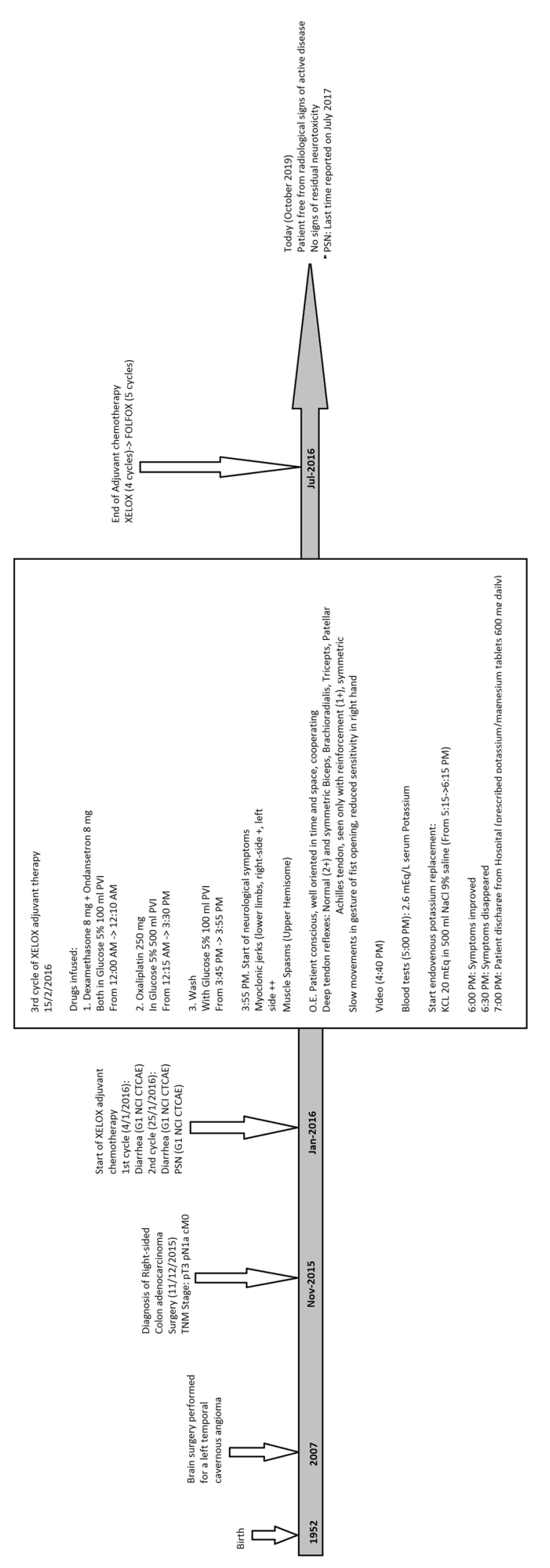

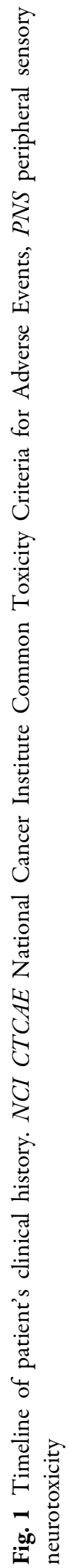


venous infusion of $20 \mathrm{mEq}$ of $\mathrm{KCl}$ in $500 \mathrm{cc}$ saline in a 1-h infusion, followed by treatment with oral potassium/magnesium tablets (600 mg per tablet, roughly estimate of $8 \mathrm{mEq}$ potassium per tablet). The patient was to continue taking the potassium/magnesium tablets after discharge from the hospital.

At approximately $2 \mathrm{~h}$ after the initiation of the potassium endovenous infusion, the symptoms slowly subsided, and after another hour the patient's condition was back to normal and he could be discharged from the hospital.

\section{Follow-Up and Outcomes}

At the fourth cycle of treatment, no motor symptoms were found during or after oxaliplatin infusion, despite the patient's complaint of sensory neuropathy in the upper limbs at the same intensity as during the previous cycles of chemotherapy. Potassium levels were around the range of normality $(3.4 \mathrm{mEq} / \mathrm{l})$ owing to the preemptive oral potassium intake before the cycle.

The patient was able to complete adjuvant treatment with the help of the oral potassium tablets (Fig. 1; Table 2), and potassium replacement was stopped at the end of the adjuvant therapy. The patient is currently free from signs of disease relapse at approximately 4 years after primary colorectal cancer surgery.

\section{DISCUSSION}

Neurotoxicity is one of the most commonly described side effects of oxaliplatin-based therapy. In this case report we describe a relatively uncommon form of neurological defect linked to oxaliplatin therapy. A retrospective assessment of the timeline of symptoms, results of laboratory tests and the outcome suggests that hypokalaemia occurring during oxaliplatin infusion could be responsible for the appearance of our patient's neurotoxicity.

As previously stated, many studies have focussed on highlighting the pathophysiology of neurological toxicity associated with oxaliplatin, and some of these have hinted at a potential role of hypokalaemia. Descoeur et al. [14] observed mice treated with oxaliplatin and
Table 2 Potassium levels before each cycle of chemotherapy

\begin{tabular}{llll}
\hline Electrolyte & $\begin{array}{l}\text { Time point of } \\
\text { measurement }\end{array}$ & $\begin{array}{l}\text { Laboratory } \\
\text { test results } \\
(\mathbf{m E q} / \mathbf{l})\end{array}$ & $\begin{array}{l}\text { Normal } \\
\text { potassium } \\
\text { range }\end{array}$ \\
\hline Potassium & Before 1st cycle & 4.2 & $3.6-4.9$ \\
& Before 2nd cycle & 3.6 & \\
Before 3rd cycle & 3.1 & \\
Before 4th cycle & 3.4 & \\
Before 5th cycle & 3.2 & \\
Before 6th cycle & 4 & \\
Before 7th cycle & 3.8 & \\
Before 8th cycle & 4.1 & \\
\hline
\end{tabular}

reported that cold hypersensitivity and hyperexcitability in these mice were associated with changes in the expression of ion channels in cold-sensing receptors. Among the receptors most affected were distinct potassium channels, such as TRAAK and TREK1, the expressions of which were lowered by oxaliplatin use, suggesting a primary role of this electrolyte in cold hypersensitivity.

Our hypothesis is that hypokalemia could be caused by the glucose infusion that is usually linked to oxaliplatin use. Oxaliplatin is always diluted in a $5 \%$ glucose solution to avoid any precipitate formation; consequently, each time oxaliplatin is infused, as much as $250-500 \mathrm{ml}$ of $5 \%$ glucose is also infused. At a high infusion speed, the sudden increase in serum glucose level is counterbalanced by an increase in circulating insulin hormone levels and, ultimately, restoration of normal glucose levels. At the same time, insulin also causes a reduction in the circulating potassium level due to an increase in uptake of both glucose and potassium by cells that are sensitive to insulin. These events may explain why our patient's hypokalaemia occurred: he was fasting during the scheduled administration of oxaliplatin and at the same time he also had slightly lower levels of potassium compared with previous cycles due to diarrhea. 
Even though we cannot exclude that oxaliplatin itself might also lower serum potassium levels directly, a series of published papers suggests that other unrelated drugs that are also diluted in glucose $5 \%$ for infusion may also cause severe hypokalaemia as a side effect; these drugs include liposomal amphotericin B, trabectedin (INN) and amiodarone. Lyposomial amphotericin B is a powerful antifungal agent used to treat systemic mycosis. The drug has to be prepared in glucose $5 \%$ prior to infusion, and one of the most common side effects is hypokalaemia $[15,16]$. This side effect of the drug is seen so frequently that preemptive treatment strategies with potassium tablets are standardly recommended. Trabectedin (INN) is a relatively novel alkylating agent used in the treatment of sarcomas and ovarian cancer. The drug has to be prepared for continuous intravenous infusion in glucose $5 \%$, and hypokalemia is reported to be one the most common side effects of the drug. In a recently published study, Jones et al. reported hypokalaemia in $25 \%$ of patients treated with trabectedin (and 5\% of patients experienced NCI CTCAE Grade 3 or higher toxicity) [17]. Interestingly amiodarone, one of the most common antiarrhythmic medications, is only soluble in glucose 5\%. Albeit the drug is used in the management of a number of arrhythmias, concomitant use of drugs that may cause hypokalaemia or a pre-existing presence of low serum potassium levels can determine the onset of severe ventricular tachycardia, such as torsade de pointes [18].

Our review of the published data on the role of electrolyte disorders due to chemotherapy and the occurrence of neurotoxicity revealed that very few case reports have been published on this subject.

Kono et al. [19] reported at least six cases of severe sensory neurotoxicity that was heightened by hypomagnesaemia caused by anti-epidermal growth factor receptor (EGFR)-based therapy in patients who were previously treated with oxaliplatin. All patients that reported residual NCI CTCAE Grade 1 peripheral neuropathy due to previous oxaliplatin exposure also reported worsening of the symptoms (up to NCI CTCAE Grade 3 neurotoxicity) whenever severe hypomagnesaemia due to using the EGRF inhibitor cetuximab. These patients suffered no motor impairment, perhaps due to the different nature of electrolytes involved in the process (magnesium vs. potassium).

Tan et al. [20] reported the case of a 47-yearold woman with metastatic gastric cancer who experienced acute motor impairment after receiving oxaliplatin-based chemotherapy. The symptoms described were due to acute bilateral abducens neuropathy, with post-tetanic facilitation along with acute laryngospasm. As in our patient, the symptoms occurred at the third cycle of treatment and were also correlated to hypokalaemia. The chemotherapy used in this case was a XELOX regimen. The "starting" serum potassium levels were already at the lower range of the normality $(3.5 \mathrm{mEq} / \mathrm{l})$, but after oxaliplatin treatment they dropped down to $2.5 \mathrm{mEq} / \mathrm{l}$, thus suggesting that hypokalaemia was the principal cause of the condition. The patient's condition improved with aggressive potassium replacement therapy, and motor impairment disappeared $2 \mathrm{~h}$ after the initiation of the potassium infusion.

Basso et al. [21] reported a case of reversible coma after a drop in both calcium and potassium levels following oxaliplatin treatment. The conditions of the patients quickly reverted to normal after normalisation of calcium and potassium levels, suggesting a primary role of electrolyte imbalance as a primary cause of the condition.

Chandar et al. [22] reported a case of a 67-year-old woman with newly diagnosed metastatic pancreatic adenocarcinoma who was treated with FOLFIRINOX. During her first infusion of irinotecan, she developed acute onset of generalised weakness, paralysis of all extremities and nonfluent aphasia with a complete inability to communicate. Prior to the infusion, her electrolyte levels were checked and found to be normal, with only mild hypokalaemia (3.5 mEq/l; normal values 3.5-5.0). The episode was self-limited and resolved within $2 \mathrm{~h}$. Prior to subsequent infusions she received intravenous repletion of potassium and had no recurrence of symptoms.

Krexner et al. [23] reported a 60-year-old female with colon cancer scheduled for a neoadjuvant chemotherapy. Five minutes before 
the end of the first oxaliplatin infusion, the patient experienced cramping of the small righthand muscles, followed by laryngospasm and bronchospasm, for which she received $250 \mathrm{mg}$ prednisolone intravenously. Despite this treatment, she developed generalised weakness, including myopathic face, severe ptosis, open mouth, ophthalmoparesis, head drop, dysphagia, dysarthria, muscular respiratory insufficiency and limb weakness, such that the patient was unable to stand or walk. Blood tests revealed hypokalaemia of 3.1 (normal range 3.7-$5.4) \mathrm{mmol} / \mathrm{l}$, hypocalcaemia of 1.98 (normal range $2.1-2.7) \mathrm{mmol} / \mathrm{l}$ and hypomagnesaemia of 0.37 (normal range 0.7-1.05) $\mathrm{mmol} / \mathrm{l}$. After about $30 \mathrm{~min}$, the acute abnormalities started to resolve. Electrolyte disturbances resolved upon adequate substitution.

These reports indicate that most of the confusion around the cause of neurotoxicity arises from the different types of electrolytes that have been evaluated as the responsible agent. To date, most studies have focussed on calcium and magnesium owing to the results of the phase II study of Grothey et al. [24] that suggested that calcium and magnesium infusions might be able to lessen the severity of neurotoxicity due to oxaliplatin-based therapy. These results were tested in the following phase III trial [25] and not validated as it was shown that the incidence of neurotoxicity was not statistically different $(p=0.972)$ among the three different treatment arms (calcium/magnesium both prior and after oxaliplatin; calcium/magnesium before oxaliplatin and placebo after oxaliplatin; placebo before and after oxaliplatin). In particular, no difference regarding both acute peripheral neuropathy and PSN could be seen.

Our case suggests that electrolyte imbalance can be the cause for the symptoms of motor impairment in patients treated with oxaliplatincontaining chemotherapy regimens. Low potassium serum levels, rather than low calcium and magnesium serum levels, seem to be associated with the majority of motor and autonomous nervous system defects. Our case is also the first in which a novel physiopathological mechanism can explain the occurrence of this electrolyte imbalance, suggesting a role of the $5 \%$ glucose dilution in electrolyte imbalances.
In their review, Oronsky et al. [26] suggested that hypokalaemia needs to be adequately managed: the pre-existing cause of hypokalaemia (bowel obstruction, diarrhea, vomiting) should be corrected, and urinary excretion of potassium should be checked so as to determine the best strategy to correct the hypokalaemia (i.e. intravenous potassium should be used only in case of arrhythmias and if metabolic acidosis is not present). Furthermore, since potassium levels are also related to magnesium levels, after assessment of magnesium levels, correction of both defects (through combined magnesium-potassium oral tablets) is to be preferred. Considering that oxaliplatin-associated neurotoxicity is only partly reversible, even after the end of treatment [27], we believe that preemptive correction of electrolyte imbalances during treatment (particularly low potassium levels) should also be advised.

\section{ACKNOWLEDGEMENTS}

We would like to thank the patient and his family for their consent to publish this study.

Funding. No funding or sponsorship was received for this study or publication of this article.

Authorship. All named authors meet the International Committee of Medical Journal Editors (ICMJE) criteria for authorship for this article, take responsibility for the integrity of the work as a whole, and have given their approval for this version to be published.

Disclosures. Elena Maccaroni, Valeria Sotte, Maria G. Baleani, Tania Meletani, Enrica Giglio, Federica Pecci, Alessandro Bittoni, Andrea Lanese, Luca Cantini, Giulia Mentrasti and Alessandra Lucarelli have nothing to disclose. Rossana Berardi is the Editor-in-Chief of this journal but has nothing else to disclose.

Compliance with Ethics Guidelines. Written informed consent for publication of this case and the patient's clinical details were asked and obtained from the patient himself and his family. 
Open Access. This article is distributed under the terms of the Creative Commons Attribution-NonCommercial 4.0 International License (http://creativecommons.org/licenses/ by-nc/4.0/), which permits any noncommercial use, distribution, and reproduction in any medium, provided you give appropriate credit to the original author(s) and the source, provide a link to the Creative Commons license, and indicate if changes were made.

\section{REFERENCES}

1. Schwartzberg LS, Rivera F, Karthaus M, et al. A randomized, multicenter phase II study of panitumumab plus modified fluorouracil, leucovorin, and oxaliplatin (mFOLFOX6) or bevacizumab plus mFOLFOX6 in patients with previously untreated, unresectable, wild-type KRAS exon 2 metastatic colorectal cancer. J Clin Oncol. 2014;32(21): 2240-7.

2. Douillard JY, Siena S, Cassidy J, et al. Final results from PRIME: randomized phase III study of panitumumab with FOLFOX4 for first-line treatment of metastatic colorectal cancer. Ann Oncol. 2014;25(7):1346-55. https://doi.org/10.1093/ annonc/mdu141. Epub 2014 Apr 8.

3. Stein A, Atanackovic D, Hildebrandt B, et al. Upfront FOLFOXIRI + bevacizumab followed by fluoropyrimidin and bevacizumab maintenance in patients with molecularly unselected metastatic colorectal cancer. Br J Cancer. 2015;113(6):872-7. https://doi.org/10.1038/bjc.2015.299. Epub 2015 Sep 3.

4. Cremolini C, Loupakis F, Antoniotti C, et al. FOLFOXIRI plus bevacizumab versus FOLFIRI plus bevacizumab as first-line treatment of patients with metastatic colorectal cancer: updated overall survival and molecular subgroup analyses of the openlabel, phase 3 TRIBE study. Lancet Oncol. 2015;16(13):1306-15. https://doi.org/10.1016/ S1470-2045(15)00122-9. Epub 2015 Aug 31.

5. Cunningham D, Starling N, Rao S, et al. Upper gastrointestinal clinical studies group of the national cancer research institute of the United Kingdom. Capecitabine and oxaliplatin for advanced esophagogastric cancer. N Engl J Med. 2008;358(1):36-46. https://doi.org/10.1056/ NEJMoa073149.

6. Conroy $\mathrm{T}$, Desseigne $\mathrm{F}$, Ychou $\mathrm{M}$, et al. FOLFIRINOX versus gemcitabine for metastatic pancreatic cancer. N Engl J Med. 2011;364(19):1817-25. https://doi.org/10.1056/NEJMoa1011923.

7. Attal N, Bouhassira D, Gautron M, et al. Thermal hyperalgesia as a marker of oxaliplatin neurotoxicity: a prospective quantified sensory assessment study. Pain. 2009;144(3):245-52. https://doi.org/ 10.1016/j.pain.2009.03.024. Epub 2009 May 19.

8. Adelsberger H, Quasthoff S, Grosskreutz J, et al. The chemotherapeutic oxaliplatin alters voltage-gated $\mathrm{Na}(+)$ channel kinetics on rat sensory neurons. Eur J Pharmacol. 2000;406:25-32.

9. Webster RG, Brain KL, Wilson RH, et al. Oxaliplatin induces hyperexcitability at motor and autonomic neuromuscular junctions through effects on voltage-gated sodium channels. $\mathrm{Br} \mathrm{J}$ Pharmacol. 2005; 146:1027-39.

10. Pereira AF, de Oliveira FFB, de Freitas Alves BW, et al. Neurotoxic effect of oxaliplatin: comparison with its oxalate-free analogue cis-[PtII(1R,2RDACH)(3-acetoxy-1,1-cyclobutanedicarboxylato)] (LLC-1402) in mice. Toxicol Appl Pharmacol. 2018;340:77-84. https://doi.org/10.1016/j.taap. 2018.01.001. Epub 2018 Jan 4.

11. Sakurai M, Egashira N, Kawashiri T, et al. Oxaliplatin-induced neuropathy in the rat: involvement of oxalate in cold hyperalgesia but not mechanical allodynia. Pain. 2009;147(1-3):165-74. https://doi. org/10.1016/j.pain.2009.09.003. Epub 2009 Sep 25.

12. Eto S, Yamamoto K, Shimazu K, et al. Formation of oxalate in oxaliplatin injection diluted with infusion solutions. Gan To Kagaku Ryoho. 2014;41(1): 71-5 (in Japanese).

13. Schmoll HJ, Tabernero J, Maroun J, et al. Capecitabine plus oxaliplatin compared with fluorouracil/folinic acid as adjuvant therapy for stage III colon cancer: final results of the NO16968 randomized controlled phase III trial. J Clin Oncol. 2015;33(32):3733-40. https://doi.org/10.1200/JCO. 2015.60.9107. Epub 2015 Aug 31.

14. Descoeur J, Pereira V, Pizzoccaro A, et al. Oxaliplatin-induced cold hypersensitivity is due to remodelling of ion channel expression in nociceptors. EMBO Mol Med. 2011;3(5):266-78. https:// doi.org/10.1002/emmm.201100134. Epub 2011 Mar 24.

15. Usami E, Kimura M, Kanematsu T, et al. Evaluation of hypokalemia and potassium supplementation during administration of liposomal-amphotericin B. Exp Ther Med. 2014;7(4):941-6.

16. Okada N, Azuma M, Imanishi M, et al. Potential usefulness of early potassium supplementation for preventing severe hypokalemia induced by 
liposomal amphotericin B in hematologic patients: a retrospective study. Clin Ther. 2018;40(2):252-60. https://doi.org/10.1016/j.clinthera.2017.12.006. Epub 2018 Jan 2.

17. Jones RL, Maki RG, Patel SR, et al. Safety and efficacy of trabectedin when administered in the inpatient versus outpatient setting: clinical considerations for outpatient administration of trabectedin. Cancer. 2019. https://doi.org/10.1002/cncr. 32462. Epub ahead of print.

18. Kotsia AP, Dimitriadis G, Baltogiannis GG, Kolettis TM. Torsade de pointes and persistent QTc prolongation after intravenous amiodarone. Case Rep Med. 2012;2012:673019. https://doi.org/10.1155/ 2012/673019. Epub 2012 Mar 5.

19. Kono T, Satomi M, Asama T, et al. Cetuximab-induced hypomagnesaemia aggravates peripheral sensory neurotoxicity caused by oxaliplatin. J Gastrointest Oncol. 2010;1(2):97-101. https://doi.org/ 10.3978/j.issn.2078-6891.2010.024.

20. Tan $\mathrm{MH}, \mathrm{Chay} \mathrm{WY}, \mathrm{Ng} \mathrm{JH}$, et al. Transient bilateral abducens neuropathy with post-tetanic facilitation and acute hypokalemia associated with oxaliplatin: a case report. J Med Case Rep. 2010;4:36. https:// doi.org/10.1186/1752-1947-4-36.

21. Basso M, Cassano A, Modoni A, et al. A reversible coma after oxaliplatin administration suggests a pathogenetic role of electrolyte imbalance. Eur J Clin Pharmacol. 2008;64(7):739-41. https://doi. org/10.1007/s00228-008-0474-X. Epub 2008 Mar 19.

22. Chandar M, De Wilton R. Severe generalized weakness, paralysis, and aphasia following administration of irinotecan and oxaliplatin during FOLFIRINOX chemotherapy. Case Rep Oncol. 2015;2015(8):138-41. https://doi.org/10.1159/ 000380849 .

23. Krexner E, Stickler A, Prainer C, Finsterer J. Acute, generalised but transient muscle cramping and weakness shortly after first oxaliplatin infusion. Med Oncol. 2012;29:3592-3. https://doi.org/10. 1007/s12032-012-0264-2.

24. Grothey A, Nikcevich DA, Sloan JA, et al. Intravenous calcium and magnesium for oxaliplatin-induced sensory neurotoxicity in adjuvant colon cancer: nCCTG N04C7. J Clin Oncol. 2011;29(4): 421-7. https://doi.org/10.1200/JCO.2010.31.5911. Epub 2010 Dec 28.

25. Loprinzi CL, Qin R, Dakhil SR, et al. Phase III randomized, placebo-controlled, double-blind study of intravenous calcium and magnesium to prevent oxaliplatin-induced sensory neurotoxicity (N08CB/ Alliance). J Clin Oncol. 2014;32(10):997-1005. https://doi.org/10.1200/JCO.2013.52.0536. Epub 2013 Dec 2.

26. Oronsky B, Caroen S, Oronsky A, et al. Electrolyte disorders with platinum-based chemotherapy: mechanisms, manifestations and management. Cancer Chemother Pharmacol. 2017;80:895-907. https://doi.org/10.1007/s00280-017-3392-8.

27. Pachman DR, Qin R, Seisler DK, et al. Clinical course of oxaliplatin-induced neuropathy: results from the randomized phase III trial NO8CB (Alliance). J Clin Oncol. 2015;33(30):3416-22. https:// doi.org/10.1200/JCO.2014.58.85330. Epub 2015 Aug 17. 